\title{
Robust Stabilization of T-S Fuzzy Systems via Improved Integral Inequality
}

\author{
C. Karthik ${ }^{a}$, G. Nagamani ${ }^{a}$,, Ramasamy Subramaniyam $^{b}$, Dafik $^{c}$ \\ ${ }^{a}$ Department of Mathematics, The Gandhigram Rural Institute (Deemed to be University), Tamil Nadu, India. \\ ${ }^{b}$ Department of Mathematics, Madanapalle Institute of Technology and Science, Andhra Pradesh, India. \\ ${ }^{c}$ Department of Mathematics Education, University of Jember, Jember-68121, Indonesia.
}

\begin{abstract}
This paper addressed the robust stabilization performance of Tagaki-Sugeno (T-S) fuzzy systems under a state feedback controller. To attain this, an integral inequality is proposed by rearranging the quadratic matrix-vector form combined with Jensen's inequality to handle the single integral terms obtained by taking the derivative of the concerned Lyapunov-Krasovskii functional (LKF). By employing this integral inequality and by using integral inequality techniques, some improved delay dependent stability and stabilization results are established in terms of linear matrix inequalities for the proposed T-S fuzzy model. Finally some numerical examples are provided to facilitate the feasibility and less conservativeness of the proposed theoretical results.
\end{abstract}

Keywords T-S fuzzy models, Time-varying delays, Linear matrix inequality, Nonlinear Systems, LyapunovKrasovskii functional.

\section{INTRODUCTION}

In the field of control engineering [1], the fuzzy logic control has been regarded as a powerful tool to anlyze the performance of the nonlinear systems. Among various fuzzy models, Takagi-Sugeno (T-S) fuzzy model is widely accepted as a simple but effective model to describe the behaviors of uncertain nonlinear systems, where a set of fuzzy IF-THEN rules is introduced to describe the local linear input-output relations of a nonlinear system 1424 . On the other hand, over the past decades, the stability and stabilization performance of uncertain T-S fuzzy systems turned out to be a more sensitive research theme [26-30]. Currently, a plenty of approaches have been developed to examine the themes of such systems, and these proposed techniques are of highly relevant to both in theory and application. For example, the problem of T-S fuzzy systems with observerbased non-parallel distributed compensation control throughout an irregular sampling interval is investigated in 25. Recently, the stabilization of T-S fuzzy model has been studied in 27] via fuzzy control approach.

In view of the practical engineering systems, there arise a couple of challenging problems such as the existence of the time-delay and the control directions probably unknown. Both always yield the control design more

\footnotetext{
${ }^{1}$ Corresponding author.

E-mail address: nagamanigru@gmail.com (G. Nagamani).
} 
complicated and complex. In such cases, the relevant LKF mechanism is always a powerful tool to deal with these shortcomings. Based on these useful techniques, many important results have been proposed through numerous inequality approaches such as Wirtinger-based inequality, weighted integral inequality, and relaxed integral inequality 28 30]. With the help of these inequalities, the derivatives of the concerned LKF are carried out to establish the desired stability conditions in terms of LMIs. To be more specific, an integral inequality proposed in 31 results in the reduction of the conservatism based on the treatment in Fourier theory, particularly in the view of well known Wirtinger inequalities. In [32], the authors developed new integral inequalities by means of the proposed integral inequality in 33 in order to investigate the problem of exponential stability performance of the delayed systems. In [34, the stability criterion for delayed system is studied with the aid of bounding inequalities and augmented LKF.

On consideration of the aforementioned issues into account, in this paper, an attempt has been made to propose an improved integral inequality to investigate the stable performance of the considered nonlinear model under fuzzy based state feedback controller. To obtain the desired stabilization criteria, an appropriate LKF has been constructed involving delay-dependent single and double integral quadratic terms. Hence, the derivative of such LKF will result in single integral terms. By introducing an appropriate dimensional matrix, the obtained single integral term can be divided into two single integral terms. Then by combining the rearrangement of Jensen's inequality and the desired quadratic matrix vector form based on the appropriate dimensioned matrix introduced earlier, a new integral inequality is proposed which yields a broad foundation for computing the single integral terms. As far as we know, the problems of stabilization for uncertain nonlinear systems via novel integral inequality have not been fully examined, which motivates us to derive the new integral inequality.

The main novelty and contribution of this manuscript can be summarized as follows:

- An improved integral inequality is acquired with help of Jensen's inequality to handle the considered LKF.

- Based on the fuzzy control approach, the stability and stabilization criteria are obtained for the considered uncertain nonlinear models through LKF whose derivative can be solved by means of the improved integral inequality.

- Appropriate control gain matrices $K_{j}$ are calculated from the LMIs to guarantee the stabilization of the proposed model via state feedback control.

- Finally, a continuous stirred-tank reactor (CSTR) system is adapted to verify the applicability and virtue of our theoretical findings. Furthermore, numerical simulations are appropriately designated to illustrate the applicability of the designed control to reduce the conservatism. 
This manuscript is organized as follows. In Section 2, we outlined the preliminaries and system descriptions. In Section 3, an improved integral inequality is introduced. Based on this integral inequality, in Section 4, the exponential stabilization of the uncertain T-S fuzzy model has been analyzed. Numerical simulation on the considered nonlinear model is provided to evaluate and illustrate the feasibility of the designed controller in Section 5. Finally, Section 6 gives the conclusion.

Notations: The notations in this manuscript are quiet standard. $A^{T}$ stands for the transpose of the matrix A. $M \geq 0(M \leq 0)$ denotes that $M$ is a positive (negative) semi-definite symmetric matrix. $\mathbb{R}^{n}$ represents an $n$-dimensional Euclidean space and $\mathbb{R}^{n \times m}$ denotes the set of all $n \times m$ real matrices. $C\left([-\tau, 0], \mathbb{R}^{n}\right)$ denotes the space of all $n$-valued continuous functions defined on $[-\tau, 0]$. A block-diagonal matrix is denoted by diag $\{\ldots\}$. For each positive integer $q$, let $\mathcal{I}_{q}=\{1,2,3, \ldots q\}$. Use $\operatorname{sym}\{M\}$ to represent $M+M^{T}$, for any matrix $M$. The symbol $0_{m \times n}$ represents null matrices of order $m \times n$. The notation $*$ is commonly used to denote the symmetric block of a symmetric matrix. I denotes the identity matrix. Matrices, if not specifically mentioned, are supposed to be appropriate dimensions.

\section{System Formulation}

We consider the following fuzzy system characterized by the fuzzy IF-THEN rules as Rule $i$ : IF $\vartheta_{1}(x(t))$ is $\mathcal{H}_{1}^{i}$, and $\cdots$, and $\vartheta_{p}(x(t))$ is $\mathcal{H}_{p}^{i}$, THEN

$$
\left\{\begin{array}{l}
\dot{x}(t)=\left[A_{i}+\Delta A_{i}(t)\right] x(t)+\left[A_{\tau_{i}}+\Delta A_{\tau_{i}}(t)\right] x(t-\tau(t))+B_{i} u(t), \\
y(t)=C x(t),
\end{array}\right.
$$

where the premise variables are represented by $\vartheta_{1}(x(t)), \vartheta_{2}(x(t)), \ldots, \vartheta_{p}(x(t))$; the fuzzy sets are denoted by $\mathcal{H}_{\varsigma}^{i},(i=1,2, \ldots, q ; \varsigma=1,2, \cdots, p), q$ represents the number of IF-THEN rules. The state vector is denoted by $x(t) \in \mathbb{R}^{n}$, the control input is represented by $u(t) \in \mathbb{R}^{m}$, the ouput vector is denoted as $y(t) \in \mathbb{R}^{v}$. The known constant system matrices are represented by $A_{i} \in \mathbb{R}^{n \times n}, A_{\tau_{i}} \in \mathbb{R}^{n \times n}, B_{i} \in \mathbb{R}^{n \times m}$ and $C \in \mathbb{R}^{n \times n}$. For system

(1), let $x(t)=\psi(t)$ be the given initial condition, where $\psi \in C\left([-\tau, 0], \mathbb{R}^{n}\right) . \tau(t)$ satisfies $0 \leq \tau(t) \leq \tau,|\dot{\tau}(t)| \leq$ $\tau_{d}<1 . \Delta A_{i}(t)$ and $\Delta A_{\tau_{i}}(t)$ denote the unknown real-valued matrices involving uncertainties of time-varying parameter defined as follows:

$$
\left[\Delta A_{i}(t) \Delta A_{\tau_{i}}(t)\right]=D_{i} F_{i}(t)\left[E_{i} E_{\tau_{i}}\right]
$$

Here $D_{i}, E_{i}$ and $E_{\tau_{i}}$ are known constant matrices and the unknown matrix $F_{i}(t)$ is follows $F_{i}^{T}(t) F_{i}(t) \leq I, \forall t$.

Then, by applying fuzzy approach, one can deduce the system dynamics (1) as follows: 


$$
\left\{\begin{array}{l}
\dot{x}(t)=\sum_{i=1}^{q} m_{i}(x(t))\left\{\left[A_{i}+\Delta A_{i}(t)\right] x(t)+\left[A_{\tau_{i}}+\Delta A_{\tau_{i}}(t)\right] x(t-\tau(t))+B_{i} u(t)\right\} \\
y(t)=C x(t)
\end{array}\right.
$$

here $m_{i}(x(t)), i=1,2, \cdots, q$, denote the normalized membership grades satisfying

$$
m_{i}(x(t))=\frac{\prod_{v=1}^{p} \mathcal{H}_{v}^{i}\left(\vartheta_{v}(x(t))\right)}{\sum_{z=1}^{q} \prod_{v=1}^{p} \mathcal{H}_{v}^{z}\left(\vartheta_{v}(x(t))\right)} \geq 0, \sum_{i=1}^{q} m_{i}(x(t))=1
$$

in which $\mathcal{H}_{v}^{i}\left(\vartheta_{v}(x(t))\right)$ is the membership grade of $\vartheta_{v}(x(t))$ subject to the fuzzy set $\mathcal{H}_{v}^{i}$.

Based on the system (2), a state feedback fuzzy model controller is designed as

Fuzzy Controller Rule $l$ : IF $\theta_{1}(x(t))$ is $\mathcal{J}_{1}^{l}$, and $\cdots$, and $\theta_{p}(x(t))$ is $\mathcal{J}_{p}^{l}$, THEN

$$
u(t)=-\sum_{l=1}^{c} w_{l}(x(t)) K_{l} x(t) .
$$

where $K_{l} \in \mathbb{R}^{m \times n},(l=1,2,3 \ldots, c)$ are control gain matrices.

Substituting (3) into (2), one can get

$$
\dot{x}(t)=\sum_{i=1}^{q} \sum_{l=1}^{c} m_{i}(x(t)) w_{l}(x(t))\left\{\left[A_{i}-B_{i} K_{l}+\Delta A_{i}(t)\right] x(t)+\left[A_{\tau_{i}}+\Delta A_{\tau_{i}}(t)\right] x(t-\tau(t))\right\} .
$$

Let us make the transformation as follows:

$$
\mathcal{M}(t)=e^{\alpha t} x(t)
$$

where $\alpha$ is decay rate of the system $(5)$.

From (5) and (4), one can get the following T-S fuzzy model:

$$
\dot{\mathcal{M}}(t)=\sum_{i=1}^{q} \sum_{l=1}^{c} m_{i}(x(t)) w_{l}(x(t))\left\{\left[\bar{A}_{i}+\Delta A_{i}(t)\right] \mathcal{M}(t)+\left[A_{\tau_{i}}+\Delta A_{\tau_{i}}(t)\right] e^{\alpha \tau} \mathcal{M}(t-\tau(t))\right\},
$$

where $\bar{A}_{i}=A_{i}-B_{i} K_{l}+\alpha I$.

\section{Improved Integral Inequality}

The flowing lemma will be derived to investigate the stability and stabilization condition of the considered nonlinear model: 
Lemma 3.1. For any symmetric matrix $L_{33}>0$ and appropriate dimensional matrices $L_{11}, L_{12}, L_{13}, L_{22}$ and $L_{23}$ satisfying $\hat{L}=\left[\begin{array}{ccc}L_{11} & L_{12} & L_{13} \\ * & L_{22} & L_{23} \\ * & * & L_{33}\end{array}\right] \geq 0$, the following inequality holds:

$$
-\int_{t-\tau(t)}^{t} \dot{x}^{T}(s) L_{33} \dot{x}(s) d s \leq \frac{1}{\tau} \zeta_{1}^{T}(t) \mathcal{E}^{T} \Lambda \mathcal{E} \zeta_{1}(t)
$$

where

$$
\begin{aligned}
& \mathcal{E}=\left[\begin{array}{l}
e_{1} \\
e_{2}
\end{array}\right], e_{i}=\left[0_{n \times(i-1) n} I 0_{n \times(2-i) n}\right], i=1,2, \Lambda=\left[\begin{array}{cc}
L_{11}+2 L_{13} & L_{12}-L_{13}+L_{22} \\
* & L_{22}-2 L_{23}
\end{array}\right], \\
& \bar{L}=\left[\begin{array}{cc}
L_{33} & -L_{33} \\
-L_{33} & L_{33}
\end{array}\right], \zeta_{1}^{T}(t)=\left[x^{T}(t) x^{T}(t-\tau(t))\right] .
\end{aligned}
$$

Proof. By applying Jensen's inequality to the integral term $-\int_{t-\tau(t)}^{t} \dot{x}^{T}(s) L_{33} \dot{x}(s) d s$, we get

$$
\begin{aligned}
-\int_{t-\tau(t)}^{t} \dot{x}^{T}(s) L_{33} \dot{x}(s) d s & \leq-\frac{1}{\tau(t)}\left(\int_{t-\tau(t)}^{t} \dot{x}(s) d s\right)^{T} L_{33}\left(\int_{t-\tau(t)}^{t} \dot{x}(s) d s\right) \\
& =-\frac{1}{\tau(t)}\left[\begin{array}{c}
x(t) \\
x(t-\tau(t))
\end{array}\right]^{T} \bar{L}\left[\begin{array}{c}
x(t) \\
x(t-\tau(t))
\end{array}\right] \\
& \leq-\frac{1}{\tau}\left[\begin{array}{c}
x(t) \\
x(t-\tau(t))
\end{array}\right]^{T} \bar{L}\left[\begin{array}{c}
x(t) \\
x(t-\tau(t))
\end{array}\right] .
\end{aligned}
$$

Also, we have the quadratic matrix vector form as

$$
\left[\begin{array}{c}
x(t) \\
x(t-\tau(t)) \\
\int_{t-\tau(t)}^{t} \dot{x}(s) d s
\end{array}\right]^{T} \hat{L}\left[\begin{array}{c}
x(t) \\
x(t-\tau(t)) \\
\int_{t-\tau(t)}^{t} \dot{x}(s) d s
\end{array}\right] \geq 0
$$

After a small computation, one can rewrite the inequality as follows:

$$
-\left[\begin{array}{c}
x(t) \\
x(t-\tau(t))
\end{array}\right]^{T} \bar{L}\left[\begin{array}{c}
x(t) \\
x(t-\tau(t))
\end{array}\right] \leq\left[\begin{array}{c}
x(t) \\
x(t-\tau(t))
\end{array}\right]^{T} \Lambda\left[\begin{array}{c}
x(t) \\
x(t-\tau(t))
\end{array}\right] .
$$


Using (8) in (7), we obtain

$$
-\int_{t-\tau(t)}^{t} \dot{x}^{T}(s) L_{33} \dot{x}(s) d s \leq \frac{1}{\tau}\left[\begin{array}{c}
x(t) \\
x(t-\tau(t))
\end{array}\right]^{T} \Lambda\left[\begin{array}{c}
x(t) \\
x(t-\tau(t))
\end{array}\right] \leq \frac{1}{\tau} \zeta_{1}^{T}(t) \mathcal{E}^{T} \Lambda \mathcal{E} \zeta_{1}(t) .
$$

Lemma 3.2. For any real matrices $D, M$, and $F(t)$ with appropriate dimensions satisfying $F^{T}(t) F(t) \leq I$, the subsequent inequality holds for any constant $\epsilon>0$ :

$$
D F(t) M+M^{T} F^{T}(t) D^{T} \leq \epsilon D D^{T}+\epsilon^{-1} M^{T} M
$$

On view of the above system configuration, the major objective of the manuscript lies in Problem 1 as follows.

Problem 1: Given the T-S fuzzy system 11, under the state feed back control, one can estimate the controller gain matrices $K_{l}, l=1,2, \ldots q$ considered in $(3)$ such that the system 6 is exponentially stable.

\section{Main Results}

In this section, for obtaining the solution of the above Problem 1, the following time-dependent LKF is considered.

$$
V(t)=\mathcal{M}^{T}(t) P \mathcal{M}(t)+\int_{t-\tau(t)}^{t} \mathcal{M}^{T}(s) Q \mathcal{M}(s) d s+\int_{-\tau}^{0} \int_{t+\theta}^{t} \dot{\mathcal{M}}^{T}(s) R \dot{\mathcal{M}}(s) d s d \theta
$$

where $P=P^{T} \in \mathbb{R}^{n \times n}, Q=Q^{T} \in \mathbb{R}^{n \times n}$, and $R=R^{T} \in \mathbb{R}^{n \times n}$ are positive definite matrices.

Theorem 4.1. For given scalars $\tau_{d}>0$ and $\tau>0$, the nominal system (6) is exponentially stable with decay rate $\alpha$, if there exist matrices $P, Q, R, M_{33}, J_{i l}, H_{i l}, i, l \in \mathcal{I}_{q}$ which are symmetric positive definite and appropriately dimensioned matrices $M_{11}, M_{12}, M_{13}, M_{22}, M_{23}$, and $K_{l} \in \mathbb{R}^{m \times n}$ under which the subsequent LMIs hold for all $i, l \in \mathcal{I}_{q}$ :

$$
\begin{gathered}
\Theta_{i l}-H_{i l}+J_{i l}+\sum_{r=1}^{q} \sum_{s=1}^{q} \bar{h}_{r s} H_{r s}-\sum_{t=1}^{q} \sum_{k=1}^{q} \underline{h}_{t k} J_{t k}<0, \\
{\left[\begin{array}{ccc}
M_{11} & M_{12} & M_{13} \\
* & M_{22} & M_{23} \\
* & * & M_{33}
\end{array}\right] \geq 0, R-M_{33} \geq 0,}
\end{gathered}
$$


where

$$
\begin{aligned}
& \underline{h}_{i l} \leq h_{i l} \leq \bar{h}_{i l}, \Theta_{i l}=\left[\begin{array}{cc}
\Theta_{i l}^{11} & \Theta_{i l}^{12} \\
* & \Theta_{i l}^{22}
\end{array}\right], \Theta_{i l}^{11}=\bar{A}_{i}^{T} P+P \bar{A}_{i}+Q+\tau \bar{A}_{i}^{T} R \bar{A}_{i}+2 M_{13}+M_{11}, \\
& \Theta_{i l}^{12}=e^{\alpha \tau} P A_{\tau_{i}}+\tau e^{\alpha \tau} \bar{A}_{i}^{T} R A_{\tau_{i}}-M_{13}+M_{23}^{T}+M_{12}, \Theta_{i l}^{22}=-\left(1-\tau_{d}\right) Q+\tau e^{2 \alpha \tau} A_{\tau_{i}}^{T} R A_{\tau_{i}}-M_{23}^{T}-M_{23}+M_{22} .
\end{aligned}
$$

Proof. Differentiating $V(t)$, one can obtain the following:

$$
\begin{aligned}
\dot{V}(t)= & 2 \dot{\mathcal{M}}^{T}(t) P \mathcal{M}(t)+\mathcal{M}^{T}(t) Q \mathcal{M}(t)-\mathcal{M}^{T}(t-\tau(t))(1-\dot{\tau}(t)) Q \mathcal{M}(t-\tau(t)) \\
& +\dot{\mathcal{M}}^{T}(t) \tau R \dot{\mathcal{M}}(t)-\int_{t-\tau}^{t} \dot{\mathcal{M}}^{T}(s) R \dot{\mathcal{M}}(s) d s \\
= & \sum_{i=1}^{q} \sum_{l=1}^{c} m_{i}(x(t)) w_{l}(x(t))\left\{\mathcal{M}^{T}(t) \bar{A}_{i}^{T} P \mathcal{M}(t)+\mathcal{M}^{T}(t) P \bar{A}_{i} \mathcal{M}(t)+\mathcal{M}^{T}(t)\left[e^{\alpha \tau} P A_{\tau_{i}}\right] \mathcal{M}(t-\tau(t))\right. \\
& \left.+\mathcal{M}^{T}(t-\tau(t))\left[e^{\alpha \tau} A_{\tau_{i}}^{T} P\right] \mathcal{M}(t)\right\}+\mathcal{M}^{T}(t) Q \mathcal{M}(t)-\mathcal{M}^{T}(t-\tau(t))(1-\dot{\tau}(t)) Q \mathcal{M}(t-\tau(t)) \\
& +\dot{\mathcal{M}}^{T}(t) \tau R \dot{\mathcal{M}}(t)-\int_{t-\tau}^{t} \dot{\mathcal{M}}^{T}(s) R \dot{\mathcal{M}}(s) d s \\
\leq & \sum_{i=1}^{q} \sum_{l=1}^{c} m_{i}(x(t)) w_{l}(x(t))\left\{\mathcal{M}^{T}(t) \bar{A}_{i}^{T} P \mathcal{M}(t)+\mathcal{M}^{T}(t) P \bar{A}_{i} \mathcal{M}(t)+\mathcal{M}^{T}(t)\left[e^{\alpha \tau} P A_{\tau_{i}}\right] \mathcal{M}(t-\tau(t))\right. \\
+ & \left.\mathcal{M}^{T}(t-\tau(t))\left[e^{\alpha \tau} A_{\tau_{i}}^{T} P\right] \mathcal{M}(t)\right\}+\mathcal{M}^{T}(t) Q \mathcal{M}(t)-\mathcal{M}^{T}(t-\tau(t))\left(1-\tau_{d}\right) Q \mathcal{M}(t-\tau(t)) \\
+ & \dot{\mathcal{M}}^{T}(t) \tau R \dot{\mathcal{M}}(t)-\int_{t-\tau(t)}^{t} \dot{\mathcal{M}}^{T}(s)\left[R-M_{33}\right] \dot{\mathcal{M}}(s) d s-\int_{t-\tau(t)}^{t} \dot{\mathcal{M}}^{T}(s) M_{33} \dot{\mathcal{M}}(s) d s .
\end{aligned}
$$

From Lemma 3.1, for any symmetric matrix $M_{33}$ and appropriate dimensioned matrices $M_{11}, M_{12}, M_{13}, M_{22}$ and $M_{23}$ satisfying $\left[\begin{array}{ccc}M_{11} & M_{12} & M_{13} \\ * & M_{22} & M_{23} \\ * & * & M_{33}\end{array}\right] \geq 0$, the following inequality can be obtained.

$$
-\int_{t-\tau(t)}^{t} \dot{\mathcal{M}}^{T}(s) M_{33} \dot{\mathcal{M}}(s) d s \leq \frac{1}{\tau} \eta^{T}(t) \mathcal{E}^{T} \Lambda_{1} \mathcal{E} \eta(t)
$$

where

$$
\Lambda_{1}=\left[\begin{array}{cc}
M_{11}+2 M_{13} & M_{12}-M_{13}+M_{22} \\
* & M_{22}-2 M_{23}
\end{array}\right], \eta^{T}(t)=\left[\begin{array}{ll}
\mathcal{M}^{T}(t) & \left.\mathcal{M}^{T}(t-\tau(t))\right] .
\end{array}\right.
$$

Adding the above inequalities (10) and [11], it follows that

$$
\dot{V}(t) \leq \sum_{i=1}^{q} \sum_{l=1}^{c} m_{i}(x(t)) w_{l}(x(t)) \eta^{T}(t) \Theta_{i l} \eta(t)-\int_{t-\tau(t)}^{t} \dot{\mathcal{M}}^{T}(s)\left[R-M_{33}\right] \dot{\mathcal{M}}(s) d s,
$$


where $\Theta_{i l}$ is defined in the Theorem 4.1. Denoting $\sum_{i=1}^{q} \sum_{l=1}^{q} m_{i}(x(t)) w_{l}(x(t))=\sum_{i=1}^{q} \sum_{l=1}^{q} h_{i l}$, by making use of some algebraic manipulations, we have

$$
\begin{aligned}
\dot{V}(t) \leq & \sum_{i=1}^{q} \sum_{l=1}^{c} h_{i l} \eta^{T}(t) \Theta_{i l} \eta(t)+\sum_{i=1}^{q} \sum_{l=1}^{c}\left[\bar{h}_{i l}-h_{i l}\right] \eta^{T}(t) H_{i l} \eta(t)+\sum_{i=1}^{q} \sum_{l=1}^{c}\left[h_{i l}-\underline{h}_{i l}\right] \eta^{T}(t) J_{i l} \eta(t) \\
& -\int_{t-\tau(t)}^{t} \dot{\mathcal{M}}^{T}(s)\left[R-M_{33}\right] \dot{\mathcal{M}}(s) d s \\
= & \sum_{i=1}^{q} \sum_{l=1}^{c} h_{i l} \eta^{T}(t)\left[\Theta_{i l}-H_{i l}+J_{i l}\right] \eta(t)+\sum_{i=1}^{q} \sum_{l=1}^{c} \bar{h}_{i l} \eta^{T}(t) H_{i l} \eta(t)-\sum_{i=1}^{q} \sum_{l=1}^{c} \underline{h}_{i l} \eta^{T}(t) J_{i l} \eta(t) \\
& -\int_{t-\tau(t)}^{t} \dot{\mathcal{M}}^{T}(s)\left[R-M_{33}\right] \dot{\mathcal{M}}(s) d s \\
= & \sum_{i=1}^{q} \sum_{l=1}^{c} h_{i l} \eta^{T}(t)\left[\Theta_{i l}-H_{i l}+J_{i l}+\sum_{r=1}^{q} \sum_{s=1}^{c} \bar{h}_{r s} H_{r s}-\sum_{j=1}^{q} \sum_{k=1}^{c} \underline{h}_{j k} J_{j k}\right] \eta(t) \\
& -\int_{t-\tau(t)}^{t} \dot{\mathcal{M}}^{T}(s)\left[R-M_{33}\right] \dot{\mathcal{M}}(s) d s,
\end{aligned}
$$

where $\bar{h}_{i l}$ is the upper bound of $h_{i l}, \underline{h}_{i l}$ is the lower bound of $h_{i l}, H_{i l}=H_{i l}^{T} \geq 0$, and $J_{i l}=J_{i l}^{T} \geq 0$. This completes the proof.

Remark 4.2. By taking $\bar{A}_{i}=A_{i}-B_{i} K_{l}+\alpha I$, the matrix $\Theta_{i l}$ can be rewritten as

$$
\begin{gathered}
\bar{\Theta}_{i l}=\left[\begin{array}{cc}
\bar{\Theta}_{i l}^{11} & \bar{\Theta}_{i l}^{12} \\
* & \bar{\Theta}_{i l}^{22}
\end{array}\right], \\
\bar{\Theta}_{i l}^{11}=\left(A_{i}-B_{i} K_{l}+\alpha I\right)^{T} P+P\left(A_{i}-B_{i} K_{l}+\alpha I\right)+Q+\tau\left(A_{i}-B_{i} K_{l}+\alpha I\right)^{T} R\left(A_{i}-B_{i} K_{l}+\alpha I\right) \\
+2 M_{13}+M_{11}, \bar{\Theta}_{i l}^{12}=e^{\alpha \tau} P A_{\tau_{i}}+\tau e^{\alpha \tau}\left(A_{i}-B_{i} K_{l}+\alpha I\right)^{T} R A_{\tau_{i}}-M_{13}+M_{23}^{T}+M_{12}, \\
\bar{\Theta}_{i l}^{22}=-\left(1-\tau_{d}\right) Q+\tau e^{2 \alpha \tau} A_{\tau_{i}}^{T} R A_{\tau_{i}}-M_{23}^{T}-M_{23}+M_{22} .
\end{gathered}
$$

Now, Theorem 4.1 can be extended to obtain the exponential stability criterion for the system (6) under the case of time-varying uncertainties as in the following theorem.

Theorem 4.3. For given scalars $\tau_{d}>0$ and $\tau>0$, the uncertain system (6) is exponentially stable with decay rate $\alpha$, if there exist matrices $P, Q, R, M_{33}, J_{i l}, H_{i l}, i, l \in \mathcal{I}_{q}$ which are symmetric positive definite and appropriately dimensioned matrices $\bar{M}_{11}, \bar{M}_{12}, \bar{M}_{13}, \bar{M}_{22}, \bar{M}_{23}$, and $K_{l} \in \mathbb{R}^{m \times n}$ under which the subsequent 
LMIs hold for all $i, l \in \mathcal{I}_{q}$ :

$$
\begin{gathered}
\Xi=\left[\begin{array}{cc}
\Xi_{i l}^{11} & \Xi_{i l}^{12} \\
* & \Xi_{i l}^{22}
\end{array}\right]<0, \\
{\left[\begin{array}{ccc}
\bar{M}_{11} & \bar{M}_{12} & \bar{M}_{13} \\
* & \bar{M}_{22} & \bar{M}_{23} \\
* & * & \bar{M}_{33}
\end{array}\right] \geq 0,} \\
\bar{R}-\bar{M}_{33} \geq 0,
\end{gathered}
$$

where $\underline{h}_{i l} \leq h_{i l} \leq \bar{h}_{i l}$

$$
\begin{aligned}
& \Xi_{i l}^{11}=\left[\begin{array}{cc}
\mathcal{M} & \Omega_{i l}^{12} \\
* & -\tau R
\end{array}\right], \Xi_{i l}^{12}=\left[\begin{array}{c}
P D_{i} \\
0 \\
\tau R D_{i}
\end{array}\right], \Xi_{i l}^{22}=-\epsilon I, \widetilde{\Theta}_{i l}=\left[\begin{array}{cc}
\widetilde{\Theta}_{i l}^{11} & \widetilde{\Theta}_{i l}^{12} \\
* & \widetilde{\Theta}_{i l}^{22}
\end{array}\right], \Omega_{i l}^{12}=\left[\begin{array}{c}
\tau \bar{A}_{i}^{T} R \\
\tau e^{\alpha \tau} A_{\tau_{i}} R
\end{array}\right], \\
& \mathcal{M}=\widetilde{\Theta}_{i l}-H_{i l}+J_{i l}+\sum_{r=1}^{q} \sum_{s=1}^{q} \bar{h}_{r s} H_{r s}-\sum_{t=1}^{q} \sum_{k=1}^{q} \underline{h}_{t k} J_{t k}, \widetilde{\Theta}_{i l}^{11}=\bar{A}_{i}^{T} P+P \bar{A}_{i}+Q+\bar{M}_{13}^{T}+\bar{M}_{13}+\bar{M}_{11}++\epsilon \mathbb{E}_{i, i}, \\
& \widetilde{\Theta}_{i l}^{12}=e^{\alpha \tau} P A_{\tau_{i}}-\bar{M}_{13}+\bar{M}_{23}^{T}+\bar{M}_{12}+\epsilon e^{\alpha \tau} \mathbb{E}_{i, \tau_{i}}, \widetilde{\Theta}_{i l}^{22}=-\left(1-\tau_{d}\right) Q-\bar{M}_{23}^{T}-\bar{M}_{23}+\bar{M}_{22}+\epsilon e^{2 \alpha \tau} \mathbb{E}_{\tau_{i}, i} .
\end{aligned}
$$

Proof. By continuing the same procedure as in Theorem 4.1 and by considering $\bar{A}_{i}=A_{i}-B_{i} K_{l}+\alpha I$, from Remark 4.2, it can be obtained that

$$
\begin{aligned}
\dot{V}(t) & \leq \sum_{i=1}^{q} \sum_{l=1}^{q} \eta^{T}(t)\left\{\bar{\Theta}_{i l}-H_{i l}+J_{i l}+\sum_{r=1}^{q} \sum_{s=1}^{q} \bar{h}_{r s} H_{r s}-\sum_{t=1}^{q} \sum_{k=1}^{q} \underline{h}_{t k} J_{t k}\right\} \eta(t) \\
& -\int_{t-\tau(t)}^{t} \dot{\mathcal{M}}^{T}(s)\left[\bar{R}-\bar{M}_{33}\right] \dot{\mathcal{M}}(s) d s .
\end{aligned}
$$

By rewriting $\bar{\Theta}_{i l}$, we obtain the following inequality:

$$
\begin{aligned}
& \dot{V}(t) \leq \sum_{i=1}^{q} \sum_{l=1}^{q} \eta^{T}(t)\left\{\left[\begin{array}{cc}
\widetilde{\Theta}_{i l}^{11} & \widetilde{\Theta}_{i l}^{12} \\
* & \widetilde{\Theta}_{i l}^{22}
\end{array}\right]+\left[\begin{array}{cc}
\tau \bar{A}_{i}^{T} R \bar{A}_{i} & \tau e^{\alpha \tau} \bar{A}_{i}^{T} R A_{\tau_{i}} \\
* & \tau e^{2 \alpha \tau} A_{\tau_{i}}^{T} R A_{\tau_{i}}
\end{array}\right]\right. \\
& \left.-H_{i l}+J_{i l}+\sum_{r=1}^{q} \sum_{s=1}^{q} h_{r s} H_{r s}-\sum_{t=1}^{q} \sum_{k=1}^{q} \underline{h}_{t k} J_{t k}\right\} \eta(t)-\int_{t-\tau(t)}^{t} \dot{\mathcal{M}}^{T}(s)\left[\bar{R}-\bar{M}_{33}\right] \dot{\mathcal{M}}(s) d s, \\
& =\sum_{i=1}^{q} \sum_{l=1}^{q} \eta^{T}(t)\left\{\left[\begin{array}{cc}
\widetilde{\Theta}_{i l}^{11} & \widetilde{\Theta}_{i l}^{12} \\
* & \widetilde{\Theta}_{i l}^{22}
\end{array}\right]+\left[\begin{array}{c}
\tau \bar{A}_{i}^{T} R \\
\tau e^{\alpha \tau} \bar{A}_{i}^{T} R
\end{array}\right][\tau R]^{-1}\left[\tau \bar{A}_{i}^{T} R \quad \tau e^{\alpha \tau} \bar{A}_{\tau i}^{T} R\right]\right.
\end{aligned}
$$




$$
\left.-H_{i l}+J_{i l}+\sum_{r=1}^{q} \sum_{s=1}^{q} h_{r s} H_{r s}-\sum_{t=1}^{q} \sum_{k=1}^{q} \underline{h}_{t k} J_{t k}\right\} \eta(t)-\int_{t-\tau(t)}^{t} \dot{\mathcal{M}}^{T}(s)\left[\bar{R}-\bar{M}_{33}\right] \dot{\mathcal{M}}(s) d s,
$$

By using the Schur complement lemma, the inequality 12 can be equivalently expressed as

$$
\left[\begin{array}{cc}
\mathcal{M} & \Omega_{i l}^{12} \\
* & -\tau R
\end{array}\right]+\epsilon^{-1}\left[\begin{array}{c}
P D_{i} \\
0 \\
\tau R D_{i}
\end{array}\right]\left[D_{i}^{T} P^{T} 0 \tau D_{i}^{T} R^{T}\right]+\epsilon\left[\begin{array}{c}
E_{i}^{T} \\
e^{\alpha \tau} E_{\tau_{i}}^{T} \\
0
\end{array}\right]\left[E_{i} e^{\alpha \tau} E_{\tau_{i}} 0\right]<0 .
$$

Hence, by Lemma 3.2 it follows that

$$
\left[\begin{array}{cc}
\mathcal{M} & \Omega_{i l}^{12} \\
* & -\tau R
\end{array}\right]+\left[\begin{array}{c}
P D_{i} \\
0 \\
\tau R D_{i}
\end{array}\right] F(t)\left[E_{i} e^{\alpha \tau} E_{\tau_{i}} 0\right]+\left[\begin{array}{c}
E_{i}^{T} \\
e^{\alpha \tau} E_{\tau_{i}}^{T} \\
0
\end{array}\right] F(t)\left[D_{i}^{T} P^{T} 0 \tau D_{i}^{T} R^{T}\right]<0
$$

Now by applying Schur complement to (16) one can obtain that

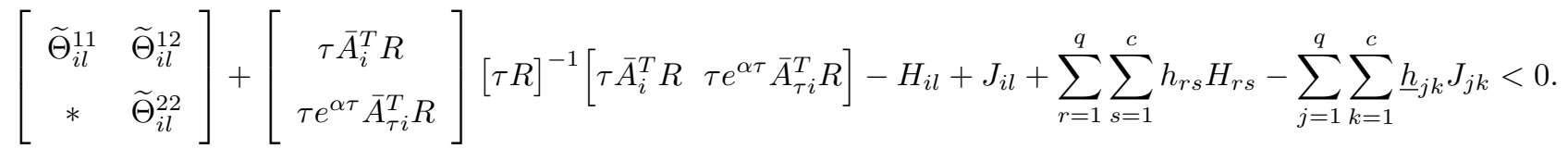

This inequality together with the inequality $\bar{R}-\bar{M}_{33} \geq 0$ implies that $\dot{V}(t)<0$. Hence the existence of the LMI (12) ensures that the exponential stability of the system (6). This completes the proof.

Remark 4.4. Based on Theorem 4.3, the gain matrices $K_{l} \in \mathbb{R}^{m \times n}\left(l \in \mathcal{I}_{q}\right)$ can be obtained by making the transformation $K_{l}=Y_{l} G^{-1},\left(l \in \mathcal{I}_{q}\right)$ as in the subsequent theorem.

Theorem 4.5. Under given scholars $a>0, \tau_{d}>0, \tau>0$ and $0<\tau(t) \leq \tau$, the system formulated in (6) is exponentially stable with decay rate $\alpha$ under the controller gain matrices $K_{l}=Y_{l} G^{-1},\left(l \in \mathcal{I}_{q}\right)$, if there exist symmetric positive definite matrices $\widehat{P}, \widehat{Q}, \widehat{R}, \widehat{M}_{33}$, and $\widehat{J}_{i l}, \widehat{H}_{i l}, i, l \in \mathcal{I}_{q}$, and appropriately dimensioned matrices $\widehat{M}_{11}, \widehat{M}_{12}, \widehat{M}_{13}, \widehat{M}_{22}, \widehat{M}_{23}$ under which the subsequent inequalities hold simultaneously for all $i, l \in \mathcal{I}_{q}$ :

$$
\begin{aligned}
& \widehat{\Xi}=\left[\begin{array}{cc}
\widehat{\Xi}_{i l}^{11} & \widehat{\Xi}_{i l}^{12} \\
* & \widehat{\Xi}_{i l}^{22}
\end{array}\right]<0, \\
& {\left[\begin{array}{ccc}
\widehat{M}_{11} & \widehat{M}_{12} & \widehat{M}_{13} \\
* & \widehat{M}_{22} & \widehat{M}_{23} \\
* & * & \widehat{M}_{33}
\end{array}\right] \geq 0} \\
& \widehat{R}-\widehat{M}_{33} \geq 0
\end{aligned}
$$


where $\underline{h}_{i l} \leq h_{i l} \leq \bar{h}_{i l}$,

$$
\begin{gathered}
\widehat{\Xi}_{i l}^{11}=\left[\begin{array}{cc}
\widehat{\mathcal{M}} & \widehat{\Omega}_{i l}^{12} \\
* & -\tau G^{T}
\end{array}\right], \widehat{\Xi}_{i l}^{12}=\left[\begin{array}{c}
D_{i} G \\
0 \\
a \tau D_{i} G
\end{array}\right], \widehat{\Xi}_{i l}^{22}=-\epsilon I, \widehat{\Theta}_{i l}=\left[\begin{array}{cc}
\widehat{\Theta}_{i l}^{11} & \widehat{\Theta}_{i l}^{12} \\
* & \widehat{\Theta}_{i l}^{22}
\end{array}\right], \\
\widehat{\Omega}_{i l}^{12}=\left[\begin{array}{c}
a \tau G^{T} A_{i}^{T}-a \tau Y_{l}^{T} B_{i}^{T}+a \alpha \tau G^{T} \\
a \tau e^{\alpha \tau} G^{T} A_{\tau_{i}}^{T}
\end{array}\right], \widehat{\mathcal{M}}=\widehat{\Theta}_{i l}-\widehat{H}_{i l} \widehat{J}_{i l}+\sum_{r=1}^{q} \sum_{s=1}^{q} \bar{h}_{r s} \widehat{H}_{r s}-\sum_{t=1}^{q} \sum_{k=1}^{q} \underline{h}_{t k} \widehat{J}_{t k} \\
\widehat{\Theta}_{i l}^{11}=G^{T} A_{i}-Y_{l}^{T} B_{i}^{T}+G^{T} \alpha+A_{i} G-B_{i} Y_{l}+\alpha G+\widehat{Q}+\widehat{M}_{33}^{T}+\widehat{M}_{33}+\widehat{M}_{11}+\epsilon \widehat{\mathbb{E}}_{i, i}, \\
\widehat{\Theta}_{i l}^{12}=e^{\alpha \tau} A_{\tau_{i}}-\widehat{M}_{13}+\widehat{M}_{23}^{T}+\widehat{M}_{12}+\epsilon e^{\alpha \tau} \widehat{\mathbb{E}}_{i, \tau_{i}}, \widehat{\Theta}_{i l}^{22}=-\left(1-\tau_{d}\right) \widehat{Q}-\widehat{M}_{23}^{T}-\widehat{M}_{23}+\widehat{M}_{22}+\epsilon e^{2 \alpha \tau} \widehat{\mathbb{E}}_{\tau_{i}, i} .
\end{gathered}
$$

Proof. Define $P=G^{-1}, R=a G^{-1}$, where $a>0$ and set $\widehat{P}=G^{T} P G, \widehat{Q}=G^{T} Q G, \widehat{R}=G^{T} R G, \quad \widehat{M}_{i j}=$ $G^{T} M_{i j} G,(i, j=1,2,3), \widehat{H}_{r s}=G^{T} H_{r s} G,(r, s=1,2,3, \ldots q), \widehat{J}_{r s}=G^{T} J_{r s} G,(r, s=1,2,3, \ldots q), \widehat{\mathbb{E}}_{i, i}=$ $G^{T} \mathbb{E}_{i, i} G,(i=1,2,3, \ldots q), \widehat{\mathbb{E}}_{\tau_{i}, i}=G^{T} \mathbb{E}_{\tau_{i}, i} G, \quad(i=1,2,3, \ldots q), \widehat{\mathbb{E}}_{i, \tau_{i}}=G^{T} \mathbb{E}_{i, \tau_{i}} G, \quad(i=1,2,3, \ldots q)$, and $Y_{l}=K_{l} G$.

Applying the congruence transformation to 12)-14 with $\operatorname{diag}\{G, G, G, G\}, \operatorname{diag}\{G, G, G\}$, and $G$ respectively, we can obtain the LMIs $177-(19)$. This completes the proof.

Remark 4.6. For facilitating the importance of the stability conditions established in this paper, we attempt to propose the less conservative stability conditions for the following system obtained from the system

$$
\dot{x}(t)=\sum_{i=1}^{q} \sum_{l=1}^{q} m_{i}(x(t)) w_{l}(x(t))\left[A_{i} x(t)+A_{\tau_{i}} x(t-\tau(t))+B_{i} u(t)\right] .
$$

obtained from the system (4) by removing the uncertain parameter matrices $\Delta A_{i}(t)$ and $\Delta A_{\tau_{i}}(t)$. From the Theorem 4.5 and by taking $\alpha=0$, the required asymptotic stability conditions for the system 20 can be acheived by adapting the following LKF:

$$
V(t)=x^{T}(t) \widetilde{P} x(t)+\int_{t-\tau(t)}^{t} x^{T}(s) \widetilde{Q} x(s) d s+\int_{-\tau}^{0} \int_{t+\theta}^{t} \dot{x}^{T}(s) \widetilde{R} \dot{x}(s) d s d \theta,
$$

where $\widetilde{P}=\widetilde{P}^{T} \in \mathbb{R}^{n \times n}, \widetilde{Q}=\widetilde{Q}^{T} \in \mathbb{R}^{n \times n}$, and $\widetilde{R}=\widetilde{R}^{T} \in \mathbb{R}^{n \times n}$ are positive definite matrices.

By making use of the same procedure as in Theorem 4.5, the following corollary can be given.

Corollary 4.7. For given scalars $a>0, \tau>0, \tau_{d}>0$ subject to $0<\tau(t) \leq \tau$, the system 20 becomes exponentially stable for the prescribed controller gain matrices $K_{l}=Y_{l} G^{-1},\left(l \in \mathcal{I}_{q}\right)$, if there exist symmetric positive definite matrices $\widetilde{P}, \widetilde{Q}, \widetilde{R}, \widetilde{M}_{33}$ and $\widetilde{J}_{r s}, \widetilde{H}_{r s}(r, s=1,2,3 \ldots q)$, and appropriately dimensioned matrices 
$\widetilde{M}_{11}, \widetilde{M}_{12}, \widetilde{M}_{13}, \widetilde{M}_{22}, \widetilde{M}_{23}$ such that the following inequalities are true for $i, l \in \mathcal{I}_{q}$ :

$$
\begin{gathered}
\widetilde{\Xi}_{i l}<0,\left[\begin{array}{ccc}
\widetilde{M}_{11} & \widetilde{M}_{12} & \widetilde{M}_{13} \\
* & \widetilde{M}_{22} & \widetilde{M}_{23} \\
* & * & \widetilde{M}_{33}
\end{array}\right] \geq 0, \widetilde{R}-\widetilde{M}_{33} \geq 0, \\
\widetilde{\Xi}_{i l}^{11}=\left[\begin{array}{cc}
\widetilde{\mathcal{M}} & \widetilde{\Omega}_{i l} \\
* & -\tau G^{T}
\end{array}\right], \widetilde{\Omega}_{i l}^{12}=\left[\begin{array}{c}
a \tau G^{T} A_{i}^{T}-a \tau Y_{l}^{T} B_{i}^{T} \\
a \tau G^{T} A_{\tau_{i}}^{T}
\end{array}\right], \\
\widetilde{\mathcal{M}}=\Gamma_{i l}-\widetilde{H}_{i l}+\widetilde{J}_{i l}+\sum_{r=1}^{q} \sum_{s=1}^{q} \bar{h}_{r s} \widetilde{H}_{r s}-\sum_{t=1}^{q} \sum_{k=1}^{q} \underline{h}_{t k} \widetilde{J}_{t k}, \Gamma_{i l}=\left[\begin{array}{cc}
\Gamma_{i l}^{11} & \Gamma_{i l}^{12} \\
* & \Gamma_{i l}^{22}
\end{array}\right], \\
\Gamma_{i l}^{11}=G^{T} A_{i}-Y_{l}^{T} B_{i}^{T}+A_{i} G-B_{i} Y_{l}+\widetilde{Q}+\widetilde{M}_{33}^{T}+\widetilde{M}_{33}+\widetilde{M}_{11}, \\
\Gamma_{i l}^{12}=A_{\tau_{i}}-\widetilde{M}_{13}+\widetilde{M}_{23}^{T}+\widetilde{M}_{12}, \Gamma_{i l}^{22}=-\left(1-\tau_{d}\right) \widetilde{Q}-\widetilde{M}_{23}^{T}-\widetilde{M}_{23}+\widetilde{M}_{22} .
\end{gathered}
$$

Proof. The proof follows immediately from Theorem 4.5 by putting $\alpha=0, \Delta A_{i}(t)=0, \Delta A_{\tau_{i}}=0$ and hence it is ignored.

\section{Numerical Examples}

Five examples are provided to demonstrate the feasibility and effectiveness of the developed control design technique in this section. In the first example, borrowed from [3, 4, a CSTR model is given to show the applicability of the considered model and the other examples are considered to show the less conservativeness of the developed technique against the conventional approaches \begin{tabular}{ll|l|l|}
35 & 38 & 42,43
\end{tabular} .

Example 5.1. Consider the following dynamic equations governing CSTR model previewed in [3], which is illustrated in Figure 1:

$$
\begin{aligned}
& \dot{x}_{1}(t)=g_{1}(x(t))+0.25 x_{1}(t-\tau), \\
& \dot{x}_{2}(t)=g_{2}(x(t))+0.25 x_{2}(t-\tau)+0.3 u(t) .
\end{aligned}
$$

where $x=\left[x_{1}(t) x_{2}(t)\right]^{T}$ in which $x_{1}$ represents the conversion rate of the reaction where as $x_{2}$ refers to the dimensionless temperature. The functions $g_{1}(x)$ and $g_{2}(x)$ are described by

$$
\begin{aligned}
& g_{1}(x)=-1.25 x_{1}(t)+0.072\left(1-x_{1}(t)\right) \exp \left(\frac{x_{2}(t)}{1+\frac{x_{2}(t)}{20}}\right), \\
& g_{2}(x)=-1.55 x_{2}(t)+0.576\left(1-x_{1}(t)\right) \exp \left(\frac{x_{2}(t)}{1+\frac{x_{2}(t)}{20}}\right) .
\end{aligned}
$$


Similar to [3], the fuzzy model of the above system are expressed under the fuzzy rules defined as follows:

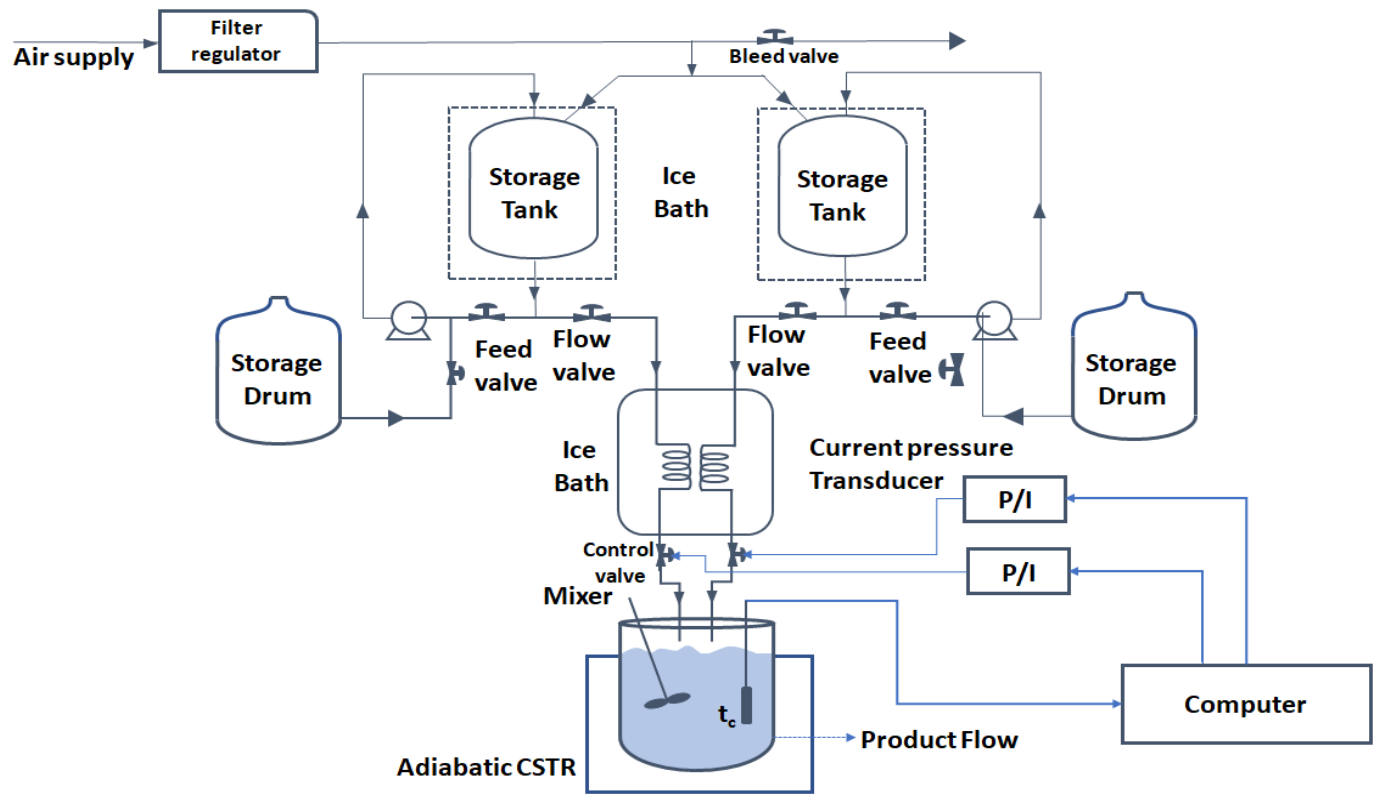

FiguRE 1. CSTR model

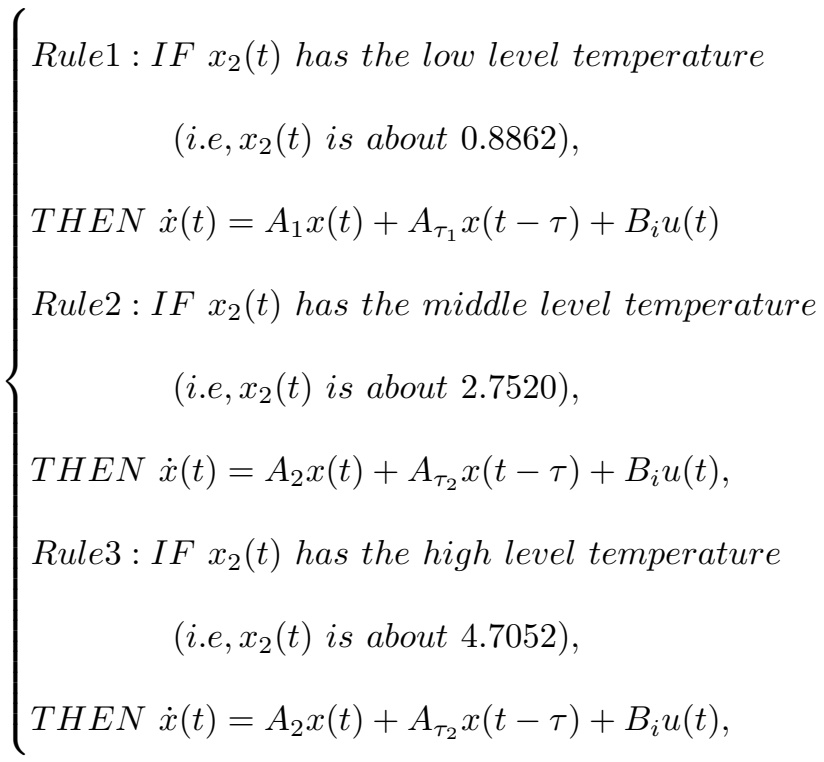

where

$$
\begin{aligned}
& A_{1}=\left[\begin{array}{cc}
-1.4274 & 0.0757 \\
-1.4189 & -0.9442
\end{array}\right], A_{\tau_{1}}=\left[\begin{array}{cc}
0.25 & 0 \\
0 & 0.25
\end{array}\right], A_{2}=\left[\begin{array}{cc}
-2.0508 & 0.3958 \\
-6.4066 & 1.6168
\end{array}\right], B_{1}=B_{2}=B_{3}=\left[\begin{array}{c}
1 \\
-1.4
\end{array}\right], \\
& A_{\tau_{2}}=\left[\begin{array}{cc}
0.25 & 0 \\
0 & 0.25
\end{array}\right], A_{3}=\left[\begin{array}{ll}
-4.5279 & 0.3167 \\
-26.2228 & 0.9837
\end{array}\right], A_{\tau_{3}}=\left[\begin{array}{cc}
0.25 & 0 \\
0 & 0.25
\end{array}\right] .
\end{aligned}
$$


Based on [3] and [5], the concerned membership functions are given as

$$
\begin{aligned}
& m_{1}\left(x_{2}(t)\right)= \begin{cases}1, & \text { if } x_{2} \leq 0.8882 \\
1-\frac{x_{2}-0.8882}{2.7520-0.8882}, & \text { if } 0.8882 \leq x_{2} \leq 2.7520 \\
0, & \text { if } x_{2} \geq 2.7520\end{cases} \\
& m_{2}\left(x_{2}(t)\right)= \begin{cases}1-m_{1}, & \text { if } x_{2} \leq 2.7520 \\
1-m_{3}, & \text { if } x_{2} \geq 2.7520\end{cases} \\
& m_{3}\left(x_{2}(t)\right)= \begin{cases}\frac{x_{2}-4.7520}{4.7520-2.7520}, & \text { if } 2.7520 \leq x_{2} \leq 4.7520 \\
1, & \text { if } x_{2} \geq 4.7520\end{cases}
\end{aligned}
$$

With respect to mismatched premise design procedure, a two-rule fuzzy controller is adapted as follows:

$$
\left\{\begin{array}{l}
\text { Rule1:IF } \theta_{1}(x(t)) i s \mathcal{J}_{1}^{1} \text {, THEN } u(t)=-K_{1} x(t) \\
\text { Rule } 2: \operatorname{IF} \theta_{1}(x(t)) i s \mathcal{J}_{1}^{2}, \text { THEN } u(t)=-K_{2} x(t)
\end{array}\right.
$$

The membership functions of fuzzy controllers are considered from [5] to be

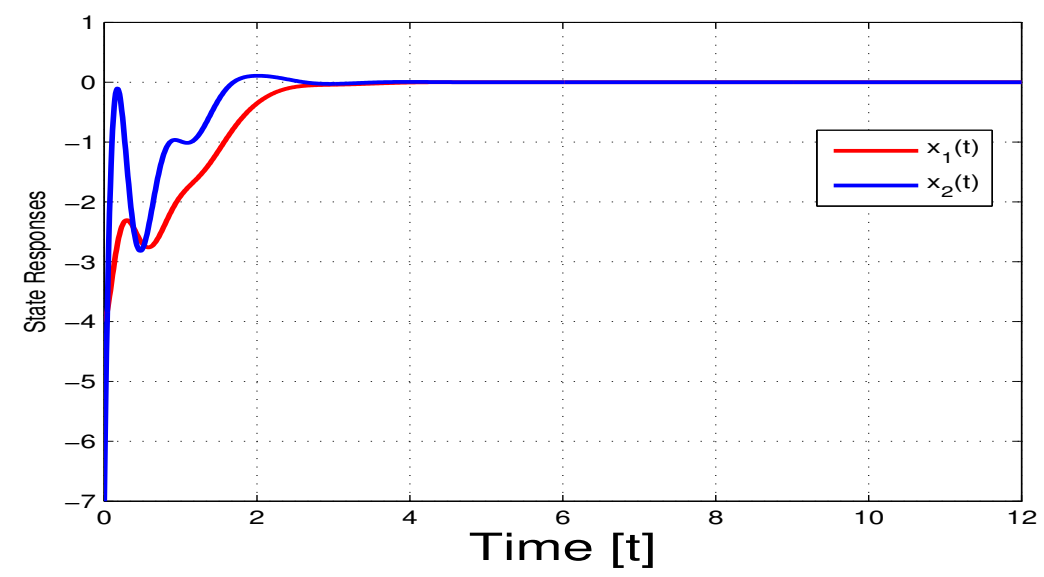

FiguRE 2. State responses of $x(t)$ for CSTR model.

$$
w_{1}\left(x_{1}\right)=\frac{1}{1+e^{-x_{1} / 2}}, w_{2}\left(x_{1}\right)=1-w_{1}\left(x_{1}\right)
$$

By fixing $\alpha=0.1, \tau=0.625, \tau_{d}=0.02$ and $\epsilon=0.01$, it is easy to show that the LMIs (17)-19) estimated in Theorem 4.5 can be made feasible by involving MATLAB LMI toolbox. This yields that the CSTR model (21) is 


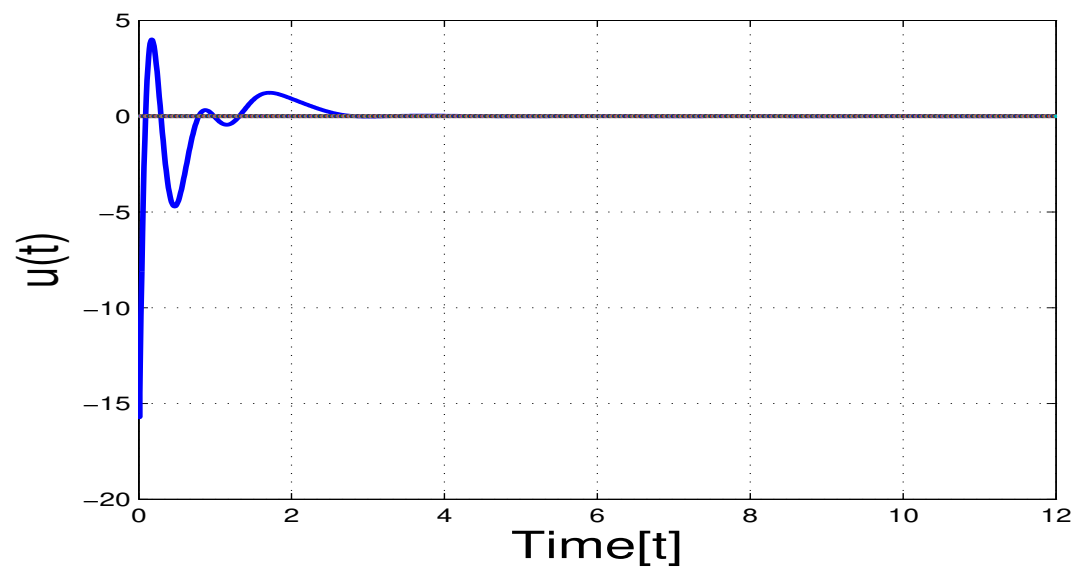

Figure 3. Control input.

TABLE 1. Determined larger delay upper bounds $\tau$ for different values of $\alpha$.

\begin{tabular}{|c|c|c|c|}
\hline$\alpha$ & 0.1 & 0.3 & 0.5 \\
\hline$\tau$ & 0.625 & 0.543 & 0.466 \\
\hline$K_{1}$ & $11.0593 \quad 4.573$ & $10.0061 \quad 4.0178$ & $10.0005 \quad 3.8272$ \\
\hline$K_{2}$ & $\begin{array}{ll}8.5066 & 3.3679\end{array}$ & 7.5103 & 7.0086 \\
\hline$K_{3}$ & 2.2369 & 4.9345 & 4.0063 \\
\hline
\end{tabular}

exponentially stable under the fuzzy controller (3) with the decay rate $\alpha=0.1$. Correspondingly, the fuzzy-based control scheme of the control gains $K_{l}(l=1,2,3)$ are calculated, as follows:

$$
K_{1}=\left[\begin{array}{ll}
1.0593 & 0.0573
\end{array}\right], K_{2}=\left[\begin{array}{ll}
1.0066 & 0.0008
\end{array}\right], K_{3}=\left[\begin{array}{ll}
1.0113 & 0.2369
\end{array}\right] .
$$

The obtained theoretical results are further examined through numerical simulation. Under the initial condition $x_{0}=\left[\begin{array}{ll}x_{1}(0) & x_{2}(0)\end{array}\right]^{T}=\left[\begin{array}{ll}-4 & -7\end{array}\right]^{T}$, by applying the fuzzy-based control developed in (3), the trajectories of the states of the considered T-S fuzzy system (21) together with the corresponding control input are depicted in Figures 2 and 3, respectively.

Example 5.2. We consider the T-S system (6) having the parameters as

$$
\begin{aligned}
& A_{1}=\left[\begin{array}{cc}
2.78 & -5.63 \\
0.01 & 0.33
\end{array}\right], A_{\tau_{1}}=\left[\begin{array}{cc}
0.5 & 0 \\
0 & -0.5
\end{array}\right], A_{2}=\left[\begin{array}{cc}
0.2 & -3.22 \\
0.35 & 0.12
\end{array}\right], A_{\tau_{2}}=\left[\begin{array}{cc}
2 & 0 \\
0 & -1
\end{array}\right], \\
& A_{3}=\left[\begin{array}{cc}
-136 & -4.33 \\
0 & 0.05
\end{array}\right], A_{\tau_{3}}=\left[\begin{array}{cc}
0.4 & 0 \\
0 & -0.6
\end{array}\right], B_{1}=\left[\begin{array}{c}
2 \\
-1
\end{array}\right], B_{2}=\left[\begin{array}{l}
8 \\
0
\end{array}\right], B_{3}=\left[\begin{array}{c}
-24 \\
-1
\end{array}\right] .
\end{aligned}
$$

Let us consider the following membership functions: 
TABLE 2. AUBs $\tau$ for various $\tau_{d}$ (Example 5.2).

\begin{tabular}{|c|c|c|}
\hline$\tau_{d}$ & 0 & 0.1 \\
\hline$[37]($ Theorem 2) & 1.6609 & 1.5332 \\
\hline $35 \sqrt{(m=3)}$ & 1.956 & 1.733 \\
\hline 42$)(m=3)$ & 2.002 & 1.8090 \\
\hline [41] & 2.0689 & 1.8447 \\
\hline [43] & 2.2943 & - \\
\hline Corollary 4.7 & 2.4906 & 1.8529 \\
\hline
\end{tabular}

$$
\begin{aligned}
& m_{1}(x(t))=1-\frac{1}{1+e^{-\left(x_{1}+4+\eta(t)\right)}}, m_{2}(x(t))=1-m_{1}(x(t))-m_{3}(x(t)), \\
& m_{3}(x(t))=1-\frac{1}{1+e^{-\left(x_{1}-4+\eta(t)\right)}} .
\end{aligned}
$$

As a result of parameter uncertainty $\eta(t)$, the membership functions designed above become the uncertain grades of membership. Therefore, the minimum and maximum membership functions are calculated as

$$
\begin{aligned}
& \underline{m}_{1}(x(t))=1-\frac{1}{1+e^{-\left(x_{1}+4+0.25\right)}}, \underline{m}_{2}(x(t))=1-\underline{m}_{1}(x(t))-\underline{m}_{3}(x(t)), \underline{m}_{3}(x(t))=1-\frac{1}{1+e^{-\left(x_{1}-4-0.25\right)}}, \\
& \bar{m}_{1}(x(t))=1-\frac{1}{1+e^{-\left(x_{1}+4-0.25\right)}}, \bar{m}_{2}(x(t))=1-\bar{m}_{1}(x(t))-\bar{m}_{3}(x(t)), \bar{m}_{3}(x(t))=1-\frac{1}{1+e^{-\left(x_{1}-4+0.25\right)}} .
\end{aligned}
$$

Let us consider the membership functions of (3) as follows:

$$
\begin{aligned}
& \underline{w}_{1}(x(t))=1-\frac{1}{1+e^{-\left(x_{1}+0.15\right) / 2}}, \underline{w}_{1}(x(t))=1-\underline{w}_{2}(x(t)), \\
& \bar{w}_{1}(x(t))=1-\frac{1}{1+e^{-\left(x_{1}-0.15\right) / 2}}, \bar{w}_{1}(x(t))=1-\bar{w}_{2}(x(t)) .
\end{aligned}
$$

With the parameters $\alpha=0.1, \tau=0.625, \tau_{d}=0.02$ and $\epsilon=0.01$, Figures 4 and 5 show that control input and the state response of the corresponding control system with the following controller gains

$$
K_{1}=\left[\begin{array}{ll}
11.0593 & 4.573
\end{array}\right], K_{2}=\left[\begin{array}{ll}
8.5066 & 3.3679
\end{array}\right], K_{3}=\left[\begin{array}{ll}
5.0113 & 2.2369
\end{array}\right] .
$$

Moreover, on solving the LMIs (17)-(19) obtained in Theorem 4.5, the corresponding larger delay upper bounds $\tau$ are calculated for different values of $\alpha$ as given in Table 1. In view of the Table 1 , one can conclude that when the value of $\tau$ decreases, simultaneously $\alpha$ increases.

The obtained theoretical results are further examined through numerical simulations. Under the initial condition $x_{0}=\left[x_{1}(0) x_{2}(0)\right]^{T}=\left[\begin{array}{ll}-4 & -7\end{array}\right]^{T}$, by applying the fuzzy-based control developed in (3), the trajectories of the states of the considered nonlinear system together with the corresponding control parameters are plotted in Figures 4 and 5, respectively. 


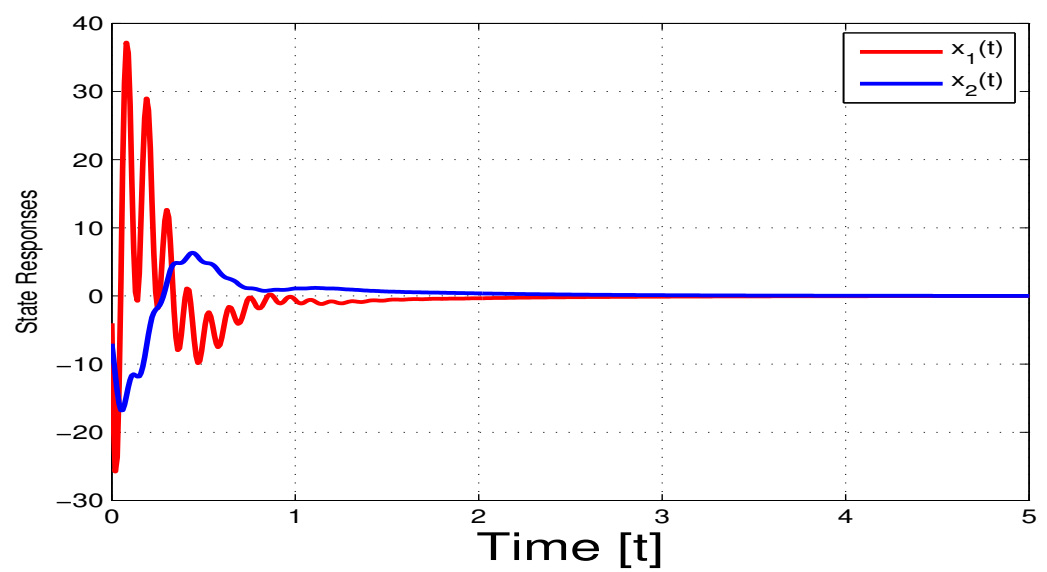

FiguRE 4. State responses of $x(t)$ for system (6).

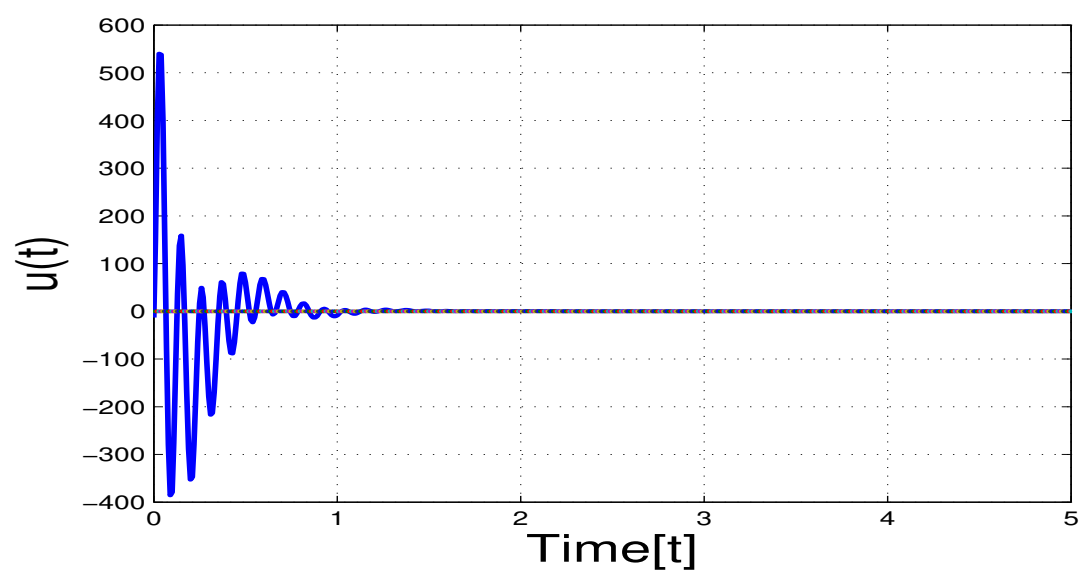

Figure 5. Control input.

Example 5.3. Consider T-S fuzzy system 201 having the subsequent parameters with two plant rule:

$$
A_{1}=\left[\begin{array}{cc}
-2 & 0 \\
0 & -0.9
\end{array}\right], A_{\tau_{1}}=\left[\begin{array}{cc}
-1 & 0 \\
-1 & -1
\end{array}\right], A_{2}=\left[\begin{array}{cc}
-1 & 0.5 \\
0 & -1
\end{array}\right], A_{\tau_{2}}=\left[\begin{array}{cc}
-1 & 0 \\
0.1 & -1
\end{array}\right] \text {. }
$$

In this example, when $\tau_{d}$ is chosen as 0 and 0.1 , the appropriate maximum values of time-delay bound $\tau$ for guaranteeing the asymptotic stability can be estimated as a result of the proposed LMIs in Corollary 4.7 and it is compared with recent literatures $37,41,43]$ as summarized in Table 2. In addition, the Corollary 4.7 provides maximum delay bounds than the existing works [35, 42] in which the delay-partitioning approach is involved. 
TABLE 3. AUBs $\tau$ for various $\tau_{d}$ (Example 5.4).

\begin{tabular}{|c|c|c|c|c|}
\hline & $\tau_{d}$ & 0 & 0.1 & 0.5 \\
\hline & {$[36]$} & 3.70 & 3.01 & 1.65 \\
\hline & [42] & 4.37 & 3.41 & 1.95 \\
\hline$[43]$ & $(m=3)$ & 5.58 & $\begin{array}{lll}- & -\end{array}$ & $\begin{array}{lll}- & - \\
\end{array}$ \\
\hline & 387 & 5.5826 & 4.2044 & 2.0685 \\
\hline Cor & llary 4.7 & 5.5796 & 4.527 & 2.079 \\
\hline
\end{tabular}

Example 5.4. Consider the the T-S fuzzy model (20) having the following system parameters with $u(t)=0$ under two plant rule:

$$
A_{1}=\left[\begin{array}{cc}
-2.1 & 0.1 \\
-0.2 & -0.9
\end{array}\right], A_{\tau_{1}}=\left[\begin{array}{cc}
-1.1 & 0.1 \\
-0.8 & -0.9
\end{array}\right], A_{2}=\left[\begin{array}{cc}
-1.9 & 0 \\
-0.2 & -1.1
\end{array}\right], A_{\tau_{2}}=\left[\begin{array}{cc}
-0.9 & 0 \\
-1.1 & -1.2
\end{array}\right] .
$$

In this example, the stability of open loop fuzzy system defined in (20) has been analyzed extremely in [18] and the goal is to calculate the larger upper bound $\tau$ with which the fuzzy system is exponentially stable. Table 3 summarizes the values of the larger upper bound $\tau$ estimated by various approaches. Through this table, it is witnessed that the performance of the developed results provides maximum delay bounds than the existing ones $36,38,42,43]$.

Example 5.5. Choose the T-S fuzzy system (20) with the parameters

$$
A_{1}=\left[\begin{array}{cc}
0 & 0.6 \\
0 & 1
\end{array}\right], A_{\tau_{1}}=\left[\begin{array}{cc}
0.5 & 0.9 \\
0 & 2
\end{array}\right], A_{2}=\left[\begin{array}{cc}
1 & 0 \\
1 & 0
\end{array}\right], A_{\tau_{2}}=\left[\begin{array}{cc}
0.9 & 0 \\
1 & 1.6
\end{array}\right], B_{1}=B_{2}=\left[\begin{array}{l}
1 \\
1
\end{array}\right] .
$$

In [38], [39], [40], and [43], the two-rule fuzzy system has been widely reviewed and the objective is to produce

TABLE 4. AUBs $\tau$ for $\tau_{d}=0$ (Example 5.5).

\begin{tabular}{|c|c|c|c|c|c|}
\hline Method & {$[40](m=3)$} & {$[39](m=3)$} & {$[43]$} & {$[38]$} & Corollary $\left[\begin{array}{l}4.7 \\
\hline \tau\end{array}\right.$ \\
\hline 1.3088 & 1.3169 & 1.3976 & 1.4212 & 1.4427 \\
\hline
\end{tabular}

the larger upper bound $\tau$ for which the fuzzy system could be stabilized by designing the appropriate controller. To obtain the less conservatism, we compare the derived results with the traditional techniques $[38,40,43]$. Table 4 gives the upper bound $\tau$ obtained by means of various approaches. Hence, it can be viewed that Corollary 4.7 establishes the higher delay bounds than the existing ones as shown in Table 4.

\section{Conclusion}

The problem of exponential stabilization for the nonlinear system via fuzzy based controller under the framework of Lyapunov approach has been investigated in this paper. First, novel integral inequality is introduced 
with the help of Jensen's inequality to limit the considered LKF. Based on the fuzzy control approach, the stability and stabilization criterion have been obtained for the considered uncertain nonlinear models through suitable LKF whose derivative can be solved via improved integral inequality. Appropriate control gain matrices have been calculated from the LMIs to guarantee the stabilization of the proposed model via state feedback control. Finally, the CSTR system has been adapted to verify the applicability and merits of our theoretical findings. Furthermore, numerical simulations have been designated appropriately to illustrate the applicability of the designed control by reducing the conservatism.

Acknowledgements None of the authors of this manuscript have a financial or personal relationship with other people or organizations that could inappropriately influence or bias the content of the manuscript.

\section{Compliance with ethical standards}

Conflict of interest It is to specifically state that "No Competing interests are at stake and there is No Conflict of Interest" with other people or organizations that could inappropriately influence or bias the content of the manuscript.

Human and animal rights This article does not contain any studies with human participants or animals performed by any of the authors.

\section{REFERENCES}

[1] T. Takagi and M. Sugeno. Fuzzy identification of systems and its applications to modeling and control. IEEE Transactions on systems, man, and cybernetics, (1):116-132, 1985.

[2] C. Lin, Q.-G. Wang, and T. H. Lee. Stabilization of uncertain fuzzy time-delay systems via variable structure control approach. IEEE Transactions on Fuzzy Systems, 13(6):787-798, 2005.

[3] C. Hua, Q.-G. Wang, and X. Guan. Robust adaptive controller design for nonlinear time-delay systems via T-S fuzzy approach. IEEE Transactions on Fuzzy Systems, 17(4):901-910, 2008.

[4] Y. Li, H.-K. Lam, L. Zhang, H. Li, F. Liu, and S.-H. Tsai. Interval type-2 fuzzy-model-based control design for time-delay systems under imperfect premise matching. In 2015 IEEE International Conference on Fuzzy Systems (FUZZ-IEEE), pages 1-6. IEEE, 2015.

[5] K. Zhou, T. Huang, T. Zhao, and F. Gao. Membership-function-dependent stability and stabilization conditions for T-S fuzzy time-delay systems. IETE Journal of Research, 65(3):351-364, 2019.

[6] S. A. Karthick, R. Sakthivel, Y.K. Ma, S. Mohanapriya, \& A. Leelamani. Disturbance rejection of fractional-order TS fuzzy neural networks based on quantized dynamic output feedback controller. Applied Mathematics and Computation, 361, 846-857, 2019 .

[7] Y. Wang, Y. Xia, and P. Zhou. Fuzzy-model-based sampled-data control of chaotic systems: A fuzzy time-dependent LyapunovKrasovskii functional approach. IEEE Transactions on fuzzy systems, 25(6):1672-1684, 2016.

[8] A. Wang, L. Liu, J. Qiu, and G. Feng. Event-triggered robust adaptive fuzzy control for a class of nonlinear systems. IEEE Transactions on Fuzzy Systems, 2018. 
[9] L. Chen, M. Liu, X. Huang, S. Fu, and J. Qiu. Adaptive fuzzy sliding mode control for network-based nonlinear systems with actuator failures. IEEE Transactions on Fuzzy Systems, 26(3):1311-1323, 2017.

[10] Y. Liu, F. Wu, and X. Ban. Dynamic output feedback control for continuous-time T-S fuzzy systems using fuzzy lyapunov functions. IEEE Transactions on Fuzzy Systems, 25(5):1155-1167, 2016.

[11] J. Qiu, G. Feng, and H. Gao. Observer-based piecewise affine output feedback controller synthesis of continuous-time T-S fuzzy affine dynamic systems using quantized measurements. IEEE Transactions on Fuzzy Systems, 20(6):1046-1062, 2012.

[12] J. Li, H. O. Wang, D. Niemann, and K. Tanaka. Dynamic parallel distributed compensation for Takagi-Sugeno fuzzy systems: an LMI approach. Information sciences, 123(3-4):201-221, 2000.

[13] M. S. Ali, G. Narayanan, V. Shekher, H. Alsulami, \& T. Saeed. Dynamic stability analysis of stochastic fractional-order memristor fuzzy BAM neural networks with delay and leakage terms. Applied Mathematics and Computation, 369, 124896, 2020 .

[14] H. Li, C. Wu, S. Yin, and H.-K. Lam. Observer-based fuzzy control for nonlinear networked systems under unmeasurable premise variables. IEEE Transactions on Fuzzy Systems, 24(5):1233-1245, 2015.

[15] R. Subramaniam, D. Song and Y. H. Joo. T-S fuzzy-based sliding mode controller design for discrete-time nonlinear model and its applications Information Sciences, 519;183-199, 2020.

[16] H. Moodi and M. Farrokhi. On observer-based controller design for Sugeno systems with unmeasurable premise variables. ISA transactions, 53(2):305-316, 2014.

[17] G. Hui and X. Xie. Novel observer-based output feedback control synthesis of discrete-time nonlinear control systems via a fuzzy approach. Neurocomputing, 214:16-22, 2016.

[18] G.-H. Yang and H. Wang. Fault detection and isolation for a class of uncertain state-feedback fuzzy control systems. IEEE Transactions on Fuzzy Systems, 23(1):139-151, 2014.

[19] Q. Gao, G. Feng, Z. Xi, Y. Wang, and J. Qiu. A new design of robust $H_{\infty}$ sliding mode control for uncertain stochastic T-S fuzzy time-delay systems. IEEE Transactions on Cybernetics, 44(9):1556-1566, 2013.

[20] R. Subramaniam and Y. H. Joo. Passivity-based fuzzy ISMC for wind energy conversion systems with PMSG. IEEE Transactions on Systems, Man, and Cybernetics: Systems., DOI: 10.1109/TSMC.2019.2930743, 2019.

[21] J. Zhang, P. Shi, J. Qiu, and S. K. Nguang. A novel observer-based output feedback controller design for discrete-time fuzzy systems. IEEE Transactions on Fuzzy Systems, 23(1):223-229, 2014.

[22] G. Nagamani and S. Ramasamy Dissipativity and passivity analysis for discrete-time T-S fuzzy stochastic neural networks with leakage time-varying delays based on Abel lemma approach. Journal of the Franklin Institute, 353(14):;313-3342, 2016.

[23] R. F. Araujo, L. A. Torres, and R. M Palhares. Distributed Control of Networked Nonlinear Systems via Interconnected TakagiSugeno Fuzzy Systems With Nonlinear Consequent. IEEE Transactions on Systems, Man, and Cybernetics: Systems, 2019.

[24] V. Nithya, R. Sakthivel, \& F. Alzahrani. Dissipative-based non-fragile filtering for fuzzy networked control systems with switching communication channels. Applied Mathematics and Computation, 373, 125011, 2020.

[25] C. Peng, S. Ma, and X. Xie. Observer-based non-PDC control for networked T-S fuzzy systems with an event-triggered communication. IEEE Transactions on Cybernetics, 47(8):2279-2287, 2017.

[26] H. Xia, H.-K. Lam, L. Li, Q. Wen, and G. Ma. Stability analysis and synthesis of fuzzy-model-based time-delay systems under imperfect premise matching. Journal of Intelligent E Fuzzy Systems, 32(6):4227-4233, 2017. 
[27] L. Wang and H.-K. Lam. A new approach to stability and stabilization analysis for continuous-time Takagi-Sugeno fuzzy systems with time delay. IEEE Transactions on Fuzzy Systems, 26(4):2460-2465, 2017.

[28] K. Gu. An integral inequality in the stability problem of time-delay systems. In Proceedings of the 39th IEEE Conference on Decision and Control (Cat. No. 00CH37187), volume 3, pages 2805-2810. IEEE, 2000.

[29] C.-K. Zhang, Y. He, L. Jiang, M. Wu, and H.-B. Zeng. Stability analysis of systems with time-varying delay via relaxed integral inequalities. Systems \& Control Letters, 92:52-61, 2016.

[30] X. Li, J. Shen, H. Akca, \& R. Rakkiyappan. LMI-based stability for singularly perturbed nonlinear impulsive differential systems with delays of small parameter. Applied Mathematics and Computation, 250, 798-804, 2015.

[31] A. Seuret and F. Gouaisbaut. Wirtinger-based integral inequality: Application to time-delay systems. Automatica, 49(9):2860$2866,2013$.

[32] C. Gong, G. Zhu, and L. Wu. New weighted integral inequalities and its application to exponential stability analysis of time-delay systems. IEEE Access, 4:6231-6237, 2016.

[33] P. Park, W. I. Lee, and S. Y. Lee. Auxiliary function-based integral inequalities for quadratic functions and their applications to time-delay systems. Journal of the Franklin Institute, 352(4):1378-1396, 2015.

[34] C.-K. Zhang, Y. He, L. Jiang, and M. Wu. Notes on stability of time-delay systems: bounding inequalities and augmented Lyapunov-Krasovskii functionals. IEEE Transactions on Automatic Control, 62(10):5331-5336, 2016.

[35] J. An and G. Wen. Improved stability criteria for time-varying delayed T-S fuzzy systems via delay partitioning approach. Fuzzy Sets and Systems, 185(1):83-94, 2011.

[36] J. An, T. Li, G. Wen, and R. Li. New stability conditions for uncertain T-S fuzzy systems with interval time-varying delay. International Journal of Control, Automation and Systems, 10(3):490-497, 2012.

[37] O. Kwon, M.-J. Park, S.-M. Lee, and J. H. Park. Augmented Lyapunov-Krasovskii functional approaches to robust stability criteria for uncertain Takagi-Sugeno fuzzy systems with time-varying delays. Fuzzy Sets and Systems, 201:1-19, 2012.

[38] O. Kwon, M.-J. Park, J. H. Park, and S.-M. Lee. Stability and stabilization of T-S fuzzy systems with time-varying delays via augmented Lyapunov-Krasovskii functionals. Information Sciences, 372:1-15, 2016.

[39] L. Mozelli, F. O. Souza, and R. Palhares. A new discretized Lyapunov-Krasovskii functional for stability analysis and control design of time-delayed T-S fuzzy systems. International Journal of Robust and Nonlinear Control, 21(1):93-105, 2011.

[40] F. O. Souza, L. A. Mozelli, and R. M. Palhares. On stability and stabilization of T-S fuzzy time-delayed systems. IEEE Transactions on Fuzzy Systems, 17(6):1450-1455, 2009.

[41] F. Yang, S. Guan, and D. Wang. Quadratically convex combination approach to stability of T-S fuzzy systems with time-varying delay. Journal of the Franklin Institute, 351(7):3752-3765, 2014.

[42] H.-B. Zeng, J. H. Park, J.-W. Xia, and S.-P. Xiao. Improved delay-dependent stability criteria for T-S fuzzy systems with time-varying delay. Applied Mathematics and Computation, 235:492-501, 2014.

[43] Z. Zhang, C. Lin, and B. Chen. New stability and stabilization conditions for T-S fuzzy systems with time delay. Fuzzy Sets and Systems, 263:82-91, 2015. 